INPLASY

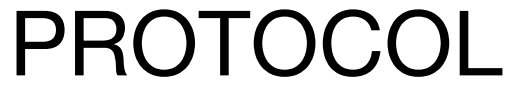

To cite: Cao et al. SodiumGlucose Cotransporter-2 Inhibitors in Heart Failure. Inplasy protocol 202180080. doi:

10.37766/inplasy2021.8.0080

Received: 21 August 2021

Published: 21 August 2021

Corresponding author:

Yi Li

doctorliyi@126.com

Author Affiliation:

The Department of Cardiology,

General Hospital of Northern

Theater Command.

Support: No.

Review Stage at time of this submission: Preliminary searches.

Conflicts of interest:

None declared.

\section{Sodium-Glucose Cotransporter-2 Inhibitors in Heart Failure}

Cao, Y1; Li, PX2; Hu, Q3; Li, Y4; Han, YL5.

Review question / Objective: What are the efficacy and safety of sodium-glucose transporter 2 inhibitors in patients with heart failure, especially heart failure with preserved ejection fraction (HFpEF)? Our aim is to evaluate the efficacy and safety of SGLT2i for heart failure regardless of LVEF and diabetes statues.

Condition being studied: SGLT2i significantly improved cardiovascular outcomes in patients with or without diabetes in several large-scale randomized controlled trials. Previous reviews are not comprehensive enough.

Study designs to be included: Compared SGLT2i versus Placebo in HF patients regardless of the left ventricular ejection fraction and diabetes statues; RCTs or its subgroup analyses and post hoc analyses; reported at least one of the predefined efficacy and safety outcomes.

INPLASY registration number: This protocol was registered with the International Platform of Registered Systematic Review and Meta-Analysis Protocols (INPLASY) on 21 August 2021 and was last updated on 21 August 2021 (registration number INPLASY202180080).

\section{INTRODUCTION}

Review question / Objective: What are the efficacy and safety of sodium-glucose transporter 2 inhibitors in patients with heart failure, especially heart failure with preserved ejection fraction (HFpEF)? Our aim is to evaluate the efficacy and safety of
SGLT2i for heart failure regardless of LVEF and diabetes statues.

Rationale: Sodium-glucose cotransporter-2 inhibitor (SGLT2i), as new effective hypoglycemic therapy, has unexpectedly shown profound cardiovascular benefits in Cardiovascular Outcomes Trials (CVOTs) 
mandated by the US Food and Drug Administration (FDA).

Condition being studied: SGLT2i significantly improved cardiovascular outcomes in patients with or without diabetes in several large-scale randomized controlled trials. Previous reviews are not comprehensive enough.

\section{METHODS}

Search strategy: The following keywords and their MeSH terms were used for the search: (sodium-glucose cotransporter-2 inhibitor OR SGLT2 inhibitor) AND (heart failure OR cardiac failure OR HF).

Participant or population: Patients with heart failure.

Intervention: Sodium-glucose cotransporter-2 inhibitor.

\section{Comparator: Placebo.}

Study designs to be included: Compared SGLT2i versus Placebo in HF patients regardless of the left ventricular ejection fraction and diabetes statues; RCTs or its subgroup analyses and post hoc analyses; reported at least one of the predefined efficacy and safety outcomes.

Eligibility criteria: Observational or nonrandomized studies; Studies that did not provide enough data to analyze the outcomes of interest.

Information sources: Mainly from electronic databases and trial registers.

Main outcome(s): Composite of first hospitalization for HF or cardiovascular death(HR); Composite of total hospitalization for HF or cardiovascular death(RR); first hospitalization for HF(HR); cardiovascular death(HR); any death(HR); major adverse cardiovascular events (HR); KCCQ(RR).

Additional outcome(s): Serious adverse events (SAE); volume depletion; hypotension; acute kidney injury; hypoglycemia; urinary tract infections; bone fracture and amputation.

Data management: Data of eligible studies will be extracted onto a predesigned excel spreadsheet.

Quality assessment / Risk of bias analysis: Quality assessment owill be conducted using Cochrane Handbook for Systematic Reviews of Interventions (version5.1.0). Publication bias will be examined using a funnel graph.

Strategy of data synthesis: Pooled hazard ratios (HRs) and 95\% confidence intervals (Cls) will be calculated for estimates of efficacy outcomes using generic inverse variance method. Dichotomous data will be used to calculate risk ratios (RRs) by Mantel-Haenszel model. The choice of fixed effects model or random effects model is made according to heterogeneity. Heterogeneity between studies was assessed using 12 statistic.

Subgroup analysis: Subgroup analyses were conducted in diabetes and nondiabetes populations.

Sensitivity analysis: Sensitivity analyses were performed by sequential trials removal.

Language: No.

Country(ies) involved: China.

Ke y words: Sodium-G Iucose Cotransporter-2 Inhibitors; Heart failure.

Contributions of each author:

Author 1 - Yang Cao.

Author 2 - Pengxiao Li.

Author 3 - Qiang Hu.

Author 4 - Yi Li.

Author 5 - Yaling Han. 